\title{
Hemp (Cannabis sativa L.) Seed Phenylpropionamides Composition and Effects on Memory Dysfunction and Biomarkers of Neuroinflammation Induced by Lipopolysaccharide in Mice
}

\author{
Yuefang Zhou, ${ }^{\dagger}$ Shanshan Wang, ${ }^{\dagger}$ Jianbo Ji, $^{\ddagger}$ Hongxiang Lou, ${ }^{\dagger}$ and Peihong Fan* ${ }^{\dagger}{ }^{\dagger}$ \\ ${ }^{\dagger}$ Department of Natural Product Chemistry, Key Lab of Chemical Biology of Ministry of Education, School of Pharmaceutical \\ Sciences and Department of Pharmacology, School of Pharmaceutical Sciences, Shandong University, Jinan 250012, China
}

Supporting Information

ABSTRACT: Hempseed has achieved a growing popularity in human nutrition, particularly regarding essential amino acids and fatty acids. The multiple positive attributes of hempseed have led to the further study of its constituents. In this study, hempseed extract containing phenylpropionamides (TPA) was obtained and its chemical profile and content were obtained using high-performance liquid chromatography technology based on previous study. The anti-neuroinflammatory effect of TPA extract was evaluated using a lipopolysaccharide (LPS)-induced mouse model. Fourteen phenylpropionamides (TPA) were identified in the obtained extract with a total content of $233.52 \pm 2.50 \mu \mathrm{g} / \mathrm{mg}$ extract. In mice, TPA prevented the learning and spatial memory damage induced by LPS. Increased brain levels of IL-1 $\beta$, IL-6, and TNF- $\alpha$ in the LPS-induced mice were reduced by TPA treatment. Furthermore, TPA attenuated LPS-induced hippocampal neuronal damage in mice. This study demonstrates the nutraceutical potential of hempseed from a neuroprotective perspective.

\section{INTRODUCTION}

Cannabis sativa L. (Cannabaceae family) has been an important source of food, fiber, and traditional medicine since historical times. ${ }^{1}$ There are two varieties that could be distinguished by the content of $\Delta^{9}$-tetrahydrocannabinol (THC), C. sativa subsp. indica (marijuana or hashish) and $C$. sativa subsp. sativa (industrial hemp). The hemp has a low content of THC, less than $0.2 \%$ on a dry basis as legal limit for the cultivation. ${ }^{2,3}$

Hempseed has been used in different way in human nutrition, including hempseed oil, ${ }^{1,4}$ milled hempseed as a source of vegetable protein, dietary fiber, ${ }^{5}$ and incorporation into food preparations (snack bars, bread, cookies, yogurt, etc). ${ }^{6}$ It is an excellent source of nutrients, especially due to its unsaturated fatty acids and essential amino acids, which are rich and in correct ratio for human dietary demands., ${ }^{4,7}$ Moreover, hempseed exert many positive effects, including alleviating constipation, ${ }^{8}$ providing cardiovascular health benefits, ${ }^{9}$ immunomodulation, and ameliorating dermatological diseases ${ }^{4}$ and gastrointestinal diseases. ${ }^{9}$ Hempseed extract showed antimutagenic effects, ${ }^{10}$ antioxidant and anti-ageing effects, ${ }^{11,12}$ and could improve chemical drugs induced learning and memory impairment in mice. ${ }^{13,14}$ It worthy noting that the ethyl acetate extract showed prominent effect among extracts prepared by different solvents (petroleum ether, ethyl acetate, $n$-butanol, and aqueous extracts). ${ }^{13}$ Thus, hempseed should be further investigated not only regarding amino acids or fatty acids but also other bioactive constituents. $^{10,15}$

Besides amino acids and fatty acids, hempseed is rich in lignanamides ${ }^{10,16}$ such as cannabisin A, B, C, etc., caffeoyltyramine-like compounds, and other polyphenols. ${ }^{10,17}$ Because the common part of these constituents is the phenylpropionamide moiety, to simplify, we refer to such constituents as phenylpropionamides in the text. In our previous study on hempseed, more than 30 phenylpropionamides were isolated. ${ }^{16,17}$ We further established a lipopolysaccharide (LPS)stimulated BV2 microglia cells model to explore the antineuroinflammatory activity of these compounds. The results showed that most lignanamides had good anti-neuroinflammatory activity by inhibiting the NF- $\kappa \mathrm{B}$ signaling pathway. ${ }^{15}$ Neuroinflammation is a critical pathological hallmark in the development of Alzheimer's disease and other neurodegenerative diseases, and high expression of inflammatory mediators in the brain is associated with $\mathrm{A} \beta$ deposition and neurofibrillary tangles. ${ }^{18}$ Lipopolysaccharide (LPS), a critical component in the outer membrane of Gram-negative bacteria, has been used as a stimulator of microglial cells and in animal models to trigger systemic and central inflammatory responses. ${ }^{19}$ Recent studies have shown that the expression

Received: September 2, 2018

Accepted: November 5, 2018

Published: November 27, 2018 


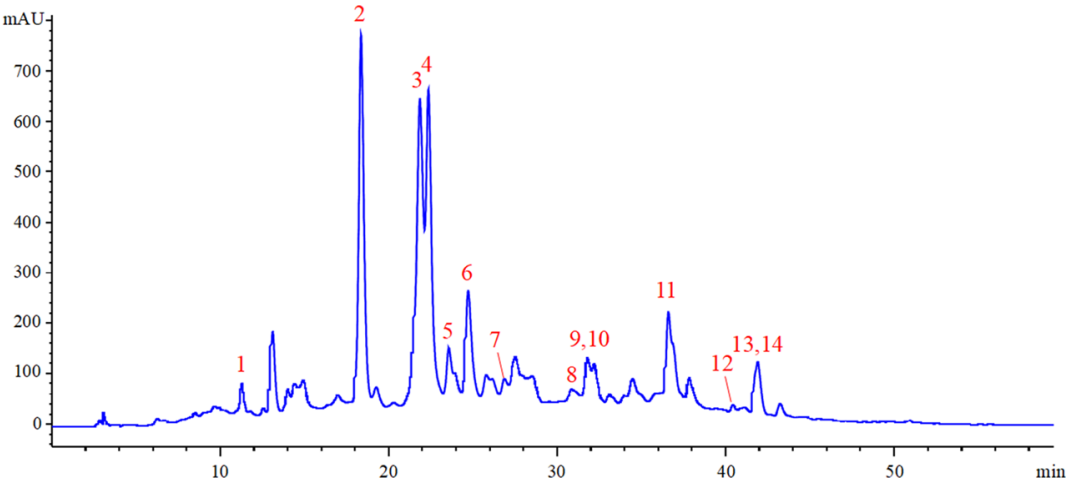

Figure 1. HPLC chromatogram of hempseed extract.

Table 1. Compounds Identified in TPA by HPLC and UV Data and Compared with Standard Compounds

\begin{tabular}{|c|c|c|c|c|c|c|}
\hline peak no. & identity & $\mathrm{RT}(\min )$ & $\mathrm{UV} \lambda_{\max }(\mathrm{nm})$ & molecular weight & molecular formula & references \\
\hline 1 & $N$-trans-caffeoyloctopamine & 11.3 & $220,290,320$ & 315 & $\mathrm{C}_{17} \mathrm{H}_{17} \mathrm{NO}_{5}$ & 17 \\
\hline 2 & $N$-trans-caffeoyltyramine & 18.4 & $220,290,320$ & 299 & $\mathrm{C}_{17} \mathrm{H}_{17} \mathrm{NO}_{4}$ & 27 \\
\hline 3 & cannabisin A & 21.8 & 255 & 594 & $\mathrm{C}_{34} \mathrm{H}_{30} \mathrm{~N}_{2} \mathrm{O}_{8}$ & 27 \\
\hline 4 & cannabisin B & 22.3 & $220,245,280,340$ & 596 & $\mathrm{C}_{34} \mathrm{H}_{32} \mathrm{~N}_{2} \mathrm{O}_{8}$ & 25 \\
\hline 5 & $N$-trans-coumaroyltyramine & 23.6 & 220,310 & 283 & $\mathrm{C}_{17} \mathrm{H}_{17} \mathrm{NO}_{3}$ & 17 \\
\hline 6 & $N$-trans-feryroyltyramine & 24.7 & $220,290,320$ & 313 & $\mathrm{C}_{18} \mathrm{H}_{19} \mathrm{NO}_{4}$ & 17 \\
\hline 8 & cannabisin D & 30.9 & $222,245,285,335$ & 624 & $\mathrm{C}_{36} \mathrm{H}_{36} \mathrm{~N}_{2} \mathrm{O}_{8}$ & 25 \\
\hline 9 & cannabisin $\mathrm{E}$ & 31.8 & $221,281,315$ & 642 & $\mathrm{C}_{36} \mathrm{H}_{38} \mathrm{~N}_{2} \mathrm{O}_{9}$ & 16 \\
\hline 10 & 3,3'-demethyl-grossamide & 32.2 & $225,285,300,320$ & 596 & $\mathrm{C}_{34} \mathrm{H}_{32} \mathrm{~N}_{2} \mathrm{O}_{8}$ & 16 \\
\hline 11 & cannabisin $\mathrm{M}$ & 36.6 & $220,285,315$ & 596 & $\mathrm{C}_{34} \mathrm{H}_{32} \mathrm{~N}_{2} \mathrm{O}_{8}$ & 16 \\
\hline 12 & isocannabisin $\mathrm{N}^{a}$ & 40.4 & $225,281,311$ & 610 & $\mathrm{C}_{35} \mathrm{H}_{34} \mathrm{~N}_{2} \mathrm{O}_{8}$ & 16 \\
\hline 13 & cannabisin $\mathrm{F}$ & 41.8 & $221,285,320$ & 624 & $\mathrm{C}_{36} \mathrm{H}_{36} \mathrm{~N}_{2} \mathrm{O}_{8}$ & 26 \\
\hline 14 & grossamide & 41.9 & $221,285,300,320$ & 624 & $\mathrm{C}_{36} \mathrm{H}_{36} \mathrm{~N}_{2} \mathrm{O}_{8}$ & 16 \\
\hline
\end{tabular}

${ }^{a}$ Compound 12 is (2,3-trans)-3-(3-hydroxy-5-methoxyphenyl)- $N$-(4-hydroxyphenethyl)-7-\{(E)-3-[(4-hydroxy phenethyl)amino]-3-oxoprop-1enyl\}-2,3-dihydro-benzo[b][1,4]dioxine-2-carboxamide; to simplify, this is referred to as isocannabisin $\mathrm{N}$.

Table 2. Peak Area and Content of TPA

\begin{tabular}{|c|c|c|c|c|c|c|}
\hline compounds & peak area & $\mu \mathrm{g} / \mathrm{mL}$ & peak area & $\mu \mathrm{g} / \mathrm{mL}$ & peak area & $\mu \mathrm{g} / \mathrm{mL}$ \\
\hline 1 & 190.1 & 4.02 & 221.8 & 4.57 & 209.3 & 4.35 \\
\hline 2 & 2190.1 & 39.03 & 2277.2 & 40.55 & 2335.4 & 41.57 \\
\hline 3 & 3024.1 & 117.13 & 3108.5 & 120.34 & 3128.2 & 121.08 \\
\hline 4 & 2472.4 & 96.20 & 2307.7 & 89.94 & 2099.0 & 82.02 \\
\hline 5 & 939.0 & 17.13 & 971.4 & 17.69 & 876.6 & 16.03 \\
\hline 6 & 1372.9 & 24.72 & 1377.3 & 24.80 & 1317.1 & 23.74 \\
\hline 7 & 419.3 & 18.28 & 408.1 & 17.85 & 365.2 & 16.22 \\
\hline 8 & 376.5 & 16.65 & 356.7 & 15.90 & 454.3 & 19.60 \\
\hline 9,10 & 870.6 & 15.93 & 891.3 & 16.29 & 854.2 & 15.64 \\
\hline 11 & 917.8 & 16.75 & 882.7 & 16.14 & 932.3 & 17.01 \\
\hline 12 & 56.6 & 1.68 & 55.5 & 1.66 & 58.9 & 1.72 \\
\hline 13,14 & 584.1 & 11.60 & 585.3 & 11.63 & 632.3 & 12.45 \\
\hline $\mathrm{TPA}(\mu \mathrm{g} / \mathrm{mL})$ & & 379.12 & & 377.36 & & 371.44 \\
\hline ТРА $(\mu \mathrm{g} / \mathrm{mg})$ & & 235.48 & & 234.38 & & 230.71 \\
\hline
\end{tabular}

of $\mathrm{A} \beta$, progressive neurodegeneration, loss of learning and memory, and the release of neuroinflammatory mediators have been detected in LPS-induced animals. ${ }^{20,21}$

The aim of this study was to investigate the antineuroinflammatory effect of hempseed extract containing mainly phenylpropionamides. The phenylpropionamide fraction was enriched from hempseed and the chemical profile of the extract was obtained using high-performance liquid chromatography (HPLC) technology based on our previous phytochemical study of hempseed. The total phenylpropana- mides (TPA) content and representative constituents were measured. The anti-neuroinflammatory effects of the TPA extract were evaluated using an LPS-induced mouse model. This study demonstrates the nutraceutical potential of hempseed from a new perspective.

\section{RESULTS}

2.1. Phytochemical Analysis of the TPA Extract of Hempseed. To obtain the TPA extract of hempseed, the dried hempseed was defatted with petroleum ether and 
A

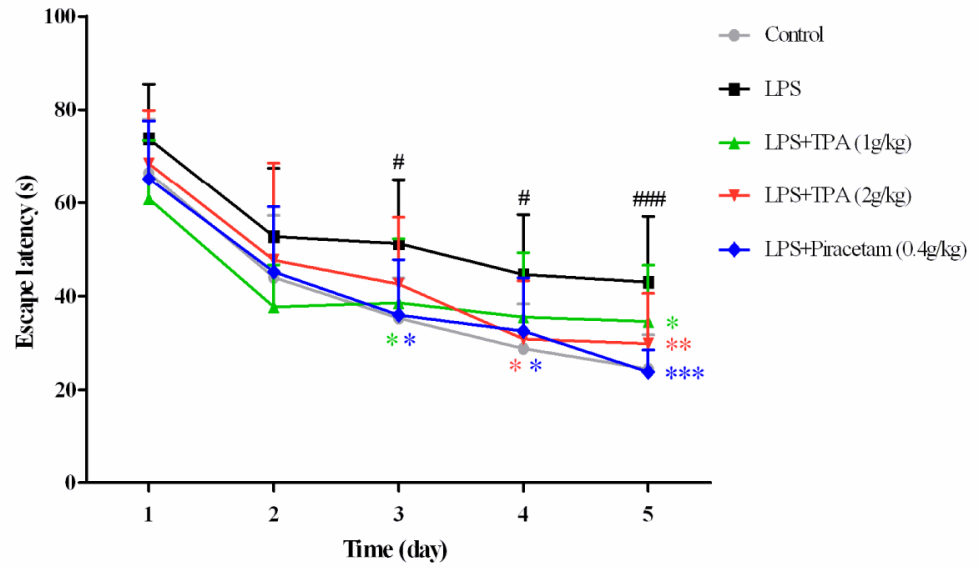

B

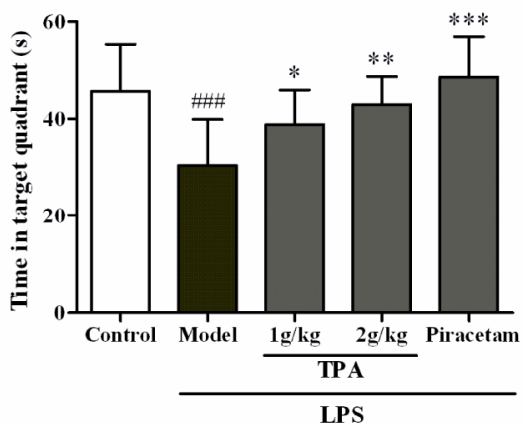

C

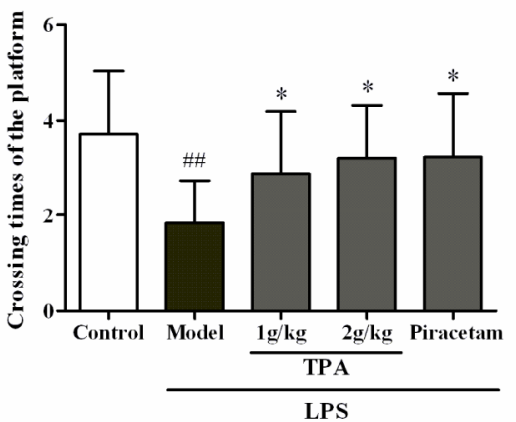

LPS+ $\mathrm{TPA}(1 \mathrm{~g} / \mathrm{kg})$
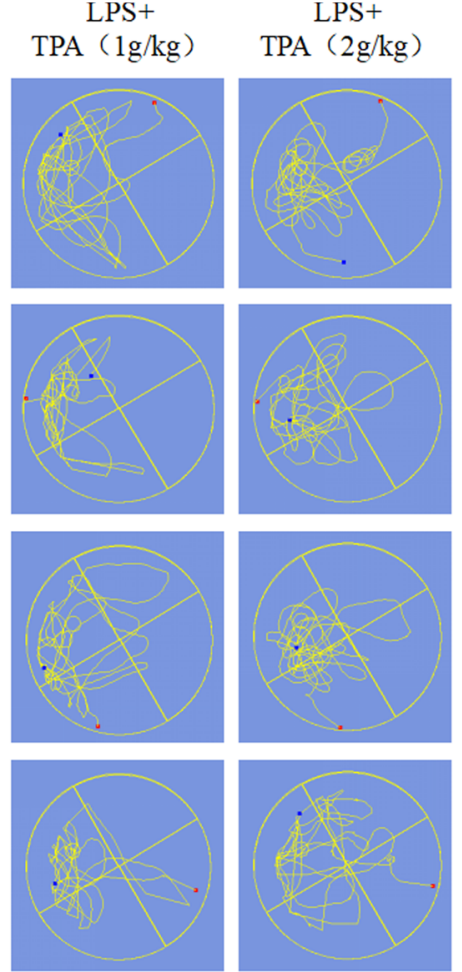

LPS+

Piracetam
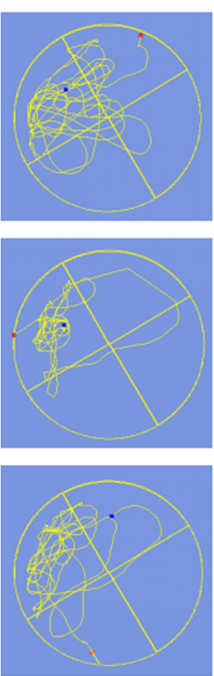

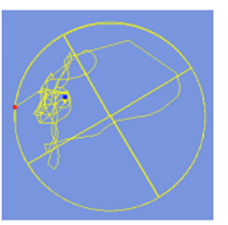

III
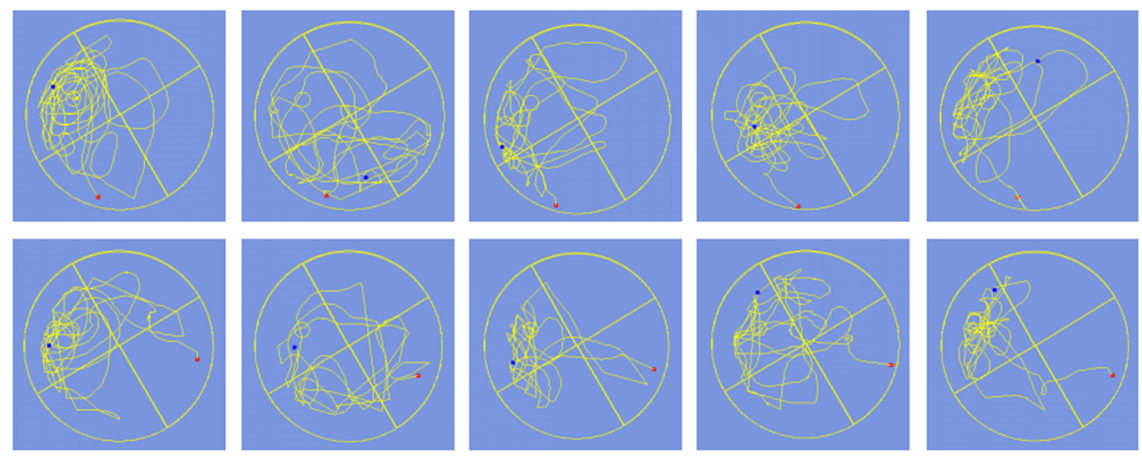

Figure 2. Effects of TPA on behavioral parameters of LPS-induced mice in the Morris water maze test $(n=12)$. (A) Escape latency during five consecutive days test. (B) Time spent in the quadrant of the platform. (C) Crossing times of the former platform location. (D) Motion trails of the mice. (Data are expressed as the mean \pm standard deviation (SD), ${ }^{\# \#} p<0.001,{ }^{\# \#} p<0.01,{ }^{\#} p<0.05$ vs control; ${ }^{* * *} p<0.001$, $* * p<0.01, * p<$ 0.05 vs LPS model.)

extracted with $70 \% \mathrm{EtOH}$. The crude extract was subjected to an D101 macroporous adsorption resin column using $\mathrm{H}_{2} \mathrm{O}$, $75 \%$, and $95 \%$ EtOH successively as eluents. Finally, the $75 \%$
EtOH fraction was collected and concentrated. HPLC technology was used to study the chemical composition of the TPA extract. Figure 1 shows the HPLC chromatogram of 
A

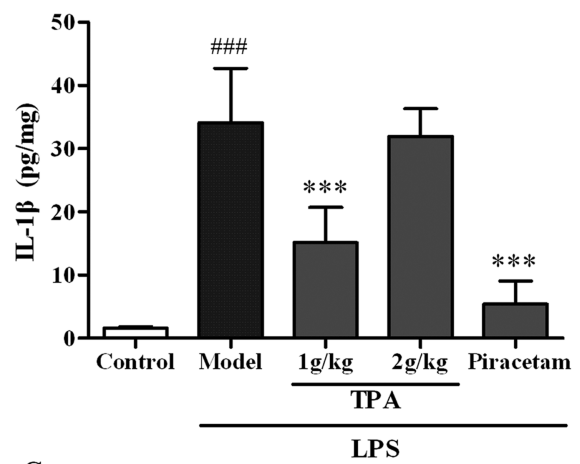

$\mathrm{C}$

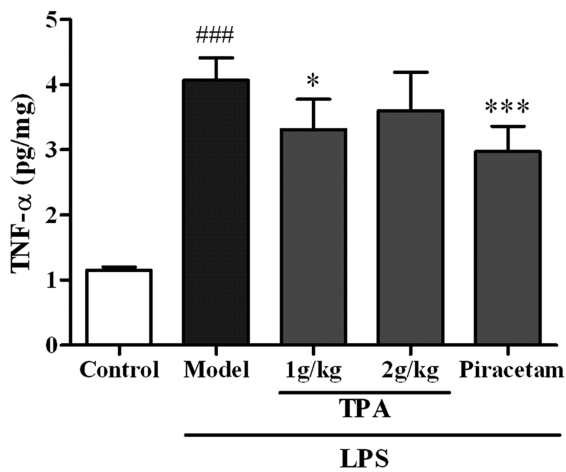

B

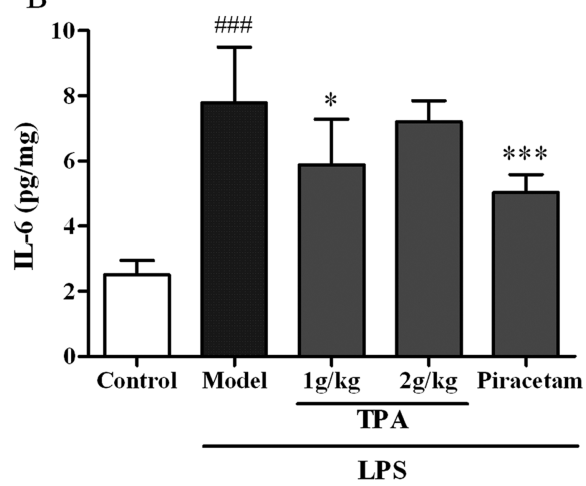

Figure 3. Effects of TPA on brain levels of pro-inflammatory cytokines in LPS-induced mice $(n=6)$ : (A) IL-1 $\beta$ level, (B) IL-6 level, and (C) TNF$\alpha$ level. (Data are expressed as the mean $\pm \mathrm{SD},{ }^{\# \# \#} p<0.001$ vs control; $* * * p<0.001$, $* p<0.05$ vs LPS model.)

the TPA extract. By combining the data from HPLC, including the retention times, the UV data on the peaks, and co-elution with previously isolated authentic samples (Figures S1-S3, Table $S 1$ in the Supporting Information), 14 phenylpropionamides were identified (Table 1, see their structures in Figure S4).

As shown in Figure 1, most compounds in the hempseed extract were identified. Therefore, the content of TPA in the extract was quantified by summing up the contents of the identified 14 compounds. Because the UV spectra of compounds 3, 4, 7, and 8 were similar to that of cannabisin A (CA) and those of the others were similar to that of N-transcaffeoyltyramine (NC), we quantified the two kinds of compounds using $\mathrm{CA}$ and $\mathrm{NC}$ as reference compounds, respectively. The standard curves of $\mathrm{CA}$ and $\mathrm{NC}$ are given in Figure S5. The content of the 14 compounds was calculated based on their measured peak area. As shown in Table 2, after summing up, the content of TPA was $233.52 \pm 2.50 \mu \mathrm{g} / \mathrm{mg}$ extract.

2.2. TPA Extract Attenuates the Cognitive Deficit Induced by LPS. Morris water maze (MWM) test was used to test the spatial learning and memory ability of mice. As shown in Figure 2A, during the five training days, the escape latency of all the groups declined progressively. This suggests that with continuous learning, the learning ability of each group increased. However, compared with the other groups, the LPS model mice spent a longer period finding the platform. Especially, on the third to fifth days, the escape latency of the model mice was significantly longer than that of the control mice ( $p<0.05, p<0.05$, and $p<0.001$, respectively). These results show that the LPS-treated mice had significant cognitive impairment. Moreover, piracetam $(0.4 \mathrm{~g} / \mathrm{kg})$-treated and TPA ( 1 and $2 \mathrm{~g} / \mathrm{kg}$ )-treated mice showed a significant shortened escape latency compared with the model mice on the fifth day $(p<0.001, p<0.05$, and $p<0.01$, respectively). In the probe trial, the platform was removed. As shown in Figure $2 \mathrm{~B}-\mathrm{D}$, the model mice spent less time $(p<0.001)$ in the target quadrant and crossed to the platform fewer times ( $p$ $<0.01)$ compared with the control group. Compared with the model group, piracetam-treated and TPA ( 1 and $2 \mathrm{~g} / \mathrm{kg}$ )treated mice showed significant increase in both the time spent in the quadrant of the platform and the number of crossing counts (both $p<0.01$ ). These results demonstrate that TPA treatment significantly improved the memory loss induced by LPS treatment in mice.

\subsection{TPA Extract Reduces Brain Levels of Inflamma-} tory Cytokines in LPS-Induced Mice. Figure 3 shows the effects of TPA extract on the brain levels of inflammatory cytokines (IL-1 $\beta$, IL-6, and TNF- $\alpha$ ) induced by LPS in mice. Enzyme-linked immunosorbent assay (ELISA) analysis indicated that exposure to LPS significantly increased the expression of IL- $1 \beta$, IL-6, and TNF- $\alpha$ in the brain compared with the control group (all $p<0.001$ ). However, pretreatment with TPA $(1 \mathrm{~g} / \mathrm{kg})$ and piracetam significantly inhibited the LPS-induced increase in IL- $1 \beta$ (both $p<0.001$, Figure 3A), IL$6(p<0.05$ and $p<0.001$, respectively, Figure 3B), and TNF- $\alpha$ $(p<0.05$ and $p<0.001$, respectively, Figure $3 \mathrm{C})$ levels in the brain in comparison to model mice, whereas TPA $(2 \mathrm{~g} / \mathrm{kg}) \mathrm{had}$ no significant effect compared with the model group. The results show that a low dose of TPA $(1 \mathrm{~g} / \mathrm{kg})$ and piracetam inhibited the overexpression of inflammatory cytokines in the brain in an LPS-induced neuroinflammation model, but a high dose of TPA may induce toxicity and counteract its role in inhibiting inflammatory cytokines production.

2.4. Protective Capacity of TPA Extract on Hippocampal Neurons. The hippocampus plays an essential role in 


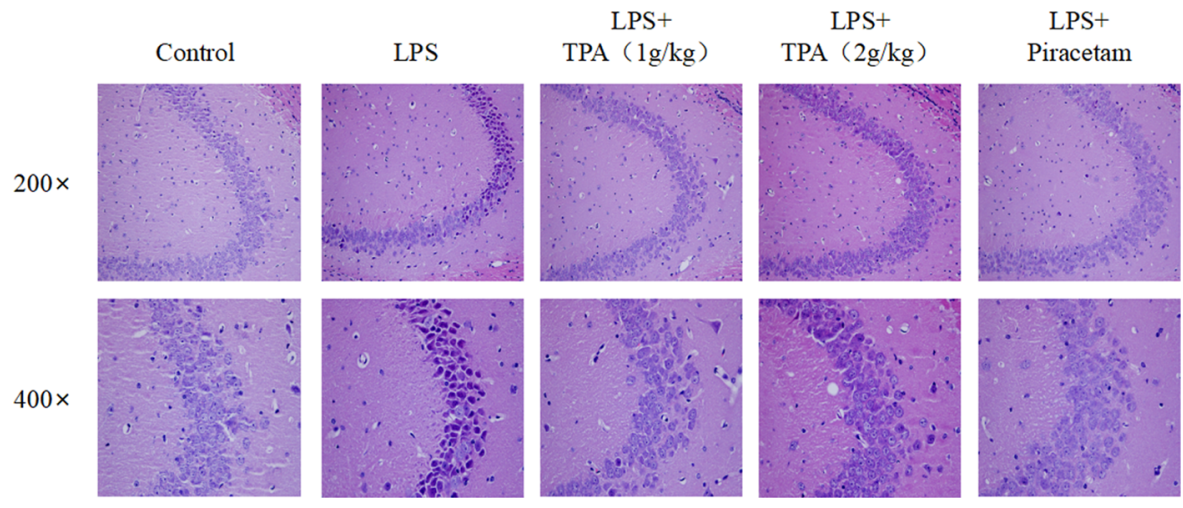

Figure 4. Effects of TPA on LPS-induced neuronal damage in hippocampal CA3 region indicated by HE staining.

spatial learning and memory function. As shown in Figure 4, hematoxylin-eosin (HE) staining indicated that the intraperitoneal injection of LPS $(250 \mu \mathrm{g} / \mathrm{kg} /$ day $)$ for 7 days caused neuronal damage. In the LPS model group, this was observed as dark-stained and deformed neurons, clearly identified in the hippocampal CA3 region. However, there were no evident neuronal morphological alterations in the TPA groups and the piracetam group, indicating that TPA and piracetam can alleviate LPS-induced neuronal damage.

\section{DISCUSSION}

The chemical constituents of hempseed are complex and diverse, including fatty acids and their esters, ${ }^{4}$ phenylpropionamides, ${ }^{16,17}$ cannabinoids, ${ }^{22}$ steroids and terpenoids, ${ }^{4}$ flavonoids and their glycosides, ${ }^{23}$ alkaloids, ${ }^{24}$ and proteins. Except fatty acids and protein, phenylpropionamides are the most studied and well-reported. Compounds of this class mainly include $N$-trans-caffeoyltyramine, $N$-trans-feryroyltyramine, and their various structural dimers called lignanamides. ${ }^{16,17,25-27}$ According to reports in the literature, more than 20 kinds of phenylpropionamides have been isolated from hempseed. ${ }^{11,26}$ Our previous study on hempseed further increased the varieties of phenylpropionamides. ${ }^{16,17}$ Some of them showed good antioxidant and anti-neuroinflammatory activities. ${ }^{16,17}$ Their bioactivities and benefits must be considered when evaluating hempseed as a functional food. Presently, studies in this field are not enough. Cai et al. ${ }^{28}$ determined the anti-aging effect of hempseed oil, protein, and ligananamide on old mice and found that all of them showed obvious effect. Lin et al. ${ }^{13}$ investigated the effect of hempseed extracts prepared by different solvents (petroleum ether, ethyl acetate, $n$-butanol and aqueous) on mice with experimental Alzheimer's disease and found that hempseed extracts conspicuously alleviated learning and memory ability, as well as improved brain tissue pathological changes in experimental dementia mice, whereas the ethyl acetate extract demonstrates a more prominent improvement than other extracts. According to our experience, ${ }^{16}$ the ethyl acetate extract contains mainly ligananamide and other phenylpropionamides. This study obtained a hempseed extract containing mainly phenylpropionamides and described its chemical profile based on our phytochemical study on hempseed. ${ }^{16,17}$ Fourteen phenylpropionamides were identified from the extract and the total content was obtained. Moreover, the neuroprotective effect of the TPA extract was evaluated using an LPS-induced neuroinflammatory model in mice.
The results showed that TPA treatment significantly improved the learning and memory of LPS-induced neuroinflammatory mice, and significantly improved their cognitive function. Low-dose TPA significantly reduced the expression of IL- $1 \beta$, IL- 6 , and TNF- $\alpha$ in the brains of LPS-induced mice. From the observation of the morphology of hippocampal neurons in mice, it was found that TPA prevented damage to nerve cells in the hippocampal CA3 region induced by LPS, indicating that TPA has neuroprotective effects. However, as we can see in the experiment, the dose-dependent relation of TPA regarding the expression of IL- $1 \beta$, IL- 6 , and TNF- $\alpha$ in the brains of LPS-induced mice was not as expected, suggesting that the safe and effective dose for TPA is less than $2 \mathrm{~g} / \mathrm{kg}$; this should be researched in more detail. According to the reports in literature, ${ }^{20,21}$ most studies use Swiss or ICR mice for modeling. Here, we have demonstrated that Kunming mice are also applicable to the LPS model. However, further studies are necessary to evaluate the anti-neuroinflammatory function of hempseed, including the use different mouse models and measuring more biomarkers.

Presently, fourteen phenylpropionamides have been identified from the extract, including caffeoyloctopamine, caffeoyltyramine, coumaroyltyramine, feryroyltyramine, cannabisins (A$\mathrm{F}, \mathrm{M}, \mathrm{N}$ ), grossamide, and demethyl-grossamide. In our previous cell-level screening test, most of them showed antineuroinflammatory activity in LPS-induced BV2 microglia cell, especially caffeoyltyramine, cannabisin F, G, M, and grossamide. ${ }^{17}$ Actually, some other phenylpropionamides of low content also showed good anti-neuroinflammatory activity, ${ }^{17}$ but they could not be identified and measured online from the extract because of their low content and lack of standard substances. We selected grossamide as a representative compound to study its anti-neuroinflammatory mechanism and found it able to significantly inhibit the LPS-mediated secretion of pro-inflammatory mediators, reduce the phosphorylation levels of NF- $\kappa \mathrm{B}$ subunit p65 in a concentrationdependent manner, and suppress the translocation of NF- $\kappa \mathrm{B}$ p65 into the nucleus, suggesting that grossamide could intervene the NF- $\kappa \mathrm{B}$ signaling pathway. ${ }^{15}$ Further study still need to be done to elucidate the anti-neuroinflammatory contribution of hempseed constituents.

Overall, our previous study found that the phenylpropanamide compounds in hempseed have significant antineuroinflammatory activities at the cellular level. In this study, we established a mouse model of neuroinflammation induced by LPS and found that hempseed extract rich in phenylpropionamides possesses effective anti-neuroinflammatory 
activity. This neuroprotective function of hempseed makes it worth noting as a functional food.

\section{MATERIALS AND METHODS}

4.1. Plant Material and Extract Preparation. Hemp ( $C$. sativa L.) seed material was collected in Bama county, Guangxi province of China, in October 2016 and identified by Professor Lan Xiang, Department of Pharmacognosy, Shandong University. Voucher specimens (201610-1) have been deposited in Dr Fan's laboratory at Shandong University, China.

The dried and crushed hempseed ( $3 \mathrm{~kg}$ ) was first defatted with petroleum ether $(25 \mathrm{~L})$ under ultrasound for $1 \mathrm{~h}$ three times and then extracted with $70 \%$ aqueous ethanol (EtOH) under reflux (three times, $25 \mathrm{~L} \times 2 \mathrm{~h}$ ). The ethanol solution was subsequently concentrated to $500 \mathrm{~mL}$ by evaporating in a vacuum. This solution was suspended in distilled water and subjected to a D101 macroporous adsorption resin column using $\mathrm{H}_{2} \mathrm{O}, 75 \%$, and $95 \% \mathrm{EtOH}$ successively to obtain the $75 \% \mathrm{EtOH}$ fraction (41.2 g, 1.37\%, extract of hempseed).

4.2. Phytochemical Identification and Quantitation of TPA in Hempseed Extract. 4.2.1. HPLC-DAD Qualitative Analysis. The $75 \% \mathrm{EtOH}$ fraction was dissolved in methanol as the sample solution and analyzed by HPLC with a diode array detector (DAD) detector. Identification of the sample solution was performed on an Agilent 1200 series HPLC system (Agilent Technologies) equipped with a G1315D DAD detector. The samples were separated through an Eclipse XDB-C18 column $\left(4.6 \times 250 \mathrm{~mm}^{2}, 5 \mu \mathrm{m}\right.$; Agilent $)$ using water (A)-acetonitrile (B) as the mobile phase, and detected under $280 \mathrm{~nm}$ at $25{ }^{\circ} \mathrm{C}$. The gradient elution for the mobile phase was set as follows: $10 \% \mathrm{~B}$ at the beginning, $20 \%$ at $5 \mathrm{~min}, 30 \%$ at $25 \mathrm{~min}, 45 \%$ at $45 \mathrm{~min}$, and $75 \%$ at $55 \mathrm{~min}$. The flow rate was $0.8 \mathrm{~mL} / \mathrm{min}$ and the injection volume was $20 \mu \mathrm{L}$.

The compounds were identified based on a comparison with the retention times and UV spectral characteristics and coelution with authentic samples previously isolated and identified by NMR analysis in our laboratory. ${ }^{16}$

4.2.2. HPLC-DAD Quantitative Analysis. To a weighed quantity containing $16.1 \mathrm{mg}$ of hempseed extract, $10 \mathrm{~mL}$ of methanol was added as the sample solution; this was repeated three times. The sample solution was analyzed according to the above-mentioned HPLC-DAD chromatographic conditions and the peak area was recorded. A standard curve was prepared using cannabisin A (CA) and N-trans-caffeoyltyramine (NC) as standard compounds, and the total content of the identified compounds was calculated.

4.3. Animals. Male Kunming mice $(31.1 \pm 2.2 \mathrm{~g}, 8$ weeks old) were obtained from Shandong University Animal Services (Shandong, China). The mice were housed under standard laboratory conditions of temperature $\left(24 \pm 1{ }^{\circ} \mathrm{C}\right)$, humidity $(50 \pm 5 \%)$, and light $(12 \mathrm{~h} \mathrm{light} /$ dark cycle $)$. The animals were allowed free access to food and water. All the procedures were approved by the Shandong University Animal Care and Use Committee and carried out in compliance with the National Institutes of Health guidelines for the care and use of laboratory animals (Publication No. 85-23, revised 1985).

4.4. Drugs and Reagents. Lipopolysaccharides (LPS) from Escherichia coli (055:B5), piracetam, and radio immunoprecipitation assay (RIPA) lysis buffer were purchased from Solarbio (Beijing, China). LPS was dissolved in $0.9 \%$ saline solution, and all the other drugs were dissolved in distilled water. TNF- $\alpha$, IL- $1 \beta$, and IL-6 enzyme-linked immunosorbent assay (ELISA) kits were purchased from Boster (Wuhan, China).

4.5. Experimental Groups and Treatment. Sixty male Kunming mice were randomly divided into five groups $(n=$ 12 /group), including control (1-3 weeks, $0.9 \%$ saline, $10 \mathrm{~mL} /$ $\mathrm{kg} /$ day), model (third week, $0.9 \%$ saline, $10 \mathrm{~mL} / \mathrm{kg} /$ day), TPA (1-3 week, 1, $2 \mathrm{~g} / \mathrm{kg} /$ day), and piracetam (2-3 week, $0.4 \mathrm{~g} /$ $\mathrm{kg} /$ day) treatment groups. Piracetam was used as a positive control due to its therapeutic effect on cognition in aging and dementia, as well as its ability to improve mitochondrial function to ameliorate the detrimental effects of $\mathrm{A} \beta$ on brain function. $^{29,30}$

After 2 weeks of administration, the model, TPA, and piracetam groups were injected intraperitoneally with LPS $(250 \mu \mathrm{g} / \mathrm{kg} /$ day $)$ on day $15,30 \mathrm{~min}$ after treatment with TPA $(1,2 \mathrm{~g} / \mathrm{kg} /$ day $)$ and piracetam $(0.4 \mathrm{~g} / \mathrm{kg} /$ day $)$. Intraperitoneal injection of LPS continued for an additional week. One hour after the LPS administration on day 16, memory function was assessed using the Morris water maze (MWM) test for 6 consecutive days (for the time line of drug treatment and experimental schedule, see Figure S6).

4.6. Morris Water Maze Test. The Morris water maze (MWM) test was used to evaluate the spatial learning and memory of the mice. ${ }^{31}$ The equipment consists of a black circular pool $(120 \mathrm{~cm}$ in diameter, $50 \mathrm{~cm}$ in height), platform, and recording system. The pool was divided into four quadrants, and an invisible transparent platform $(9 \mathrm{~cm}$ in diameter) was placed at the center of the second quadrant and submerged $1 \mathrm{~cm}$ below the water surface. A black edible pigment was added to the water, so the water was opaque. The water temperature was maintained at $22-24{ }^{\circ} \mathrm{C}$. This behavioral task included hidden-platform training (spatial learning) and probe trial (spatial memory) sessions.

In the hidden-platform training session, the mice were allowed four daily trials in the presence of the platform for five subsequent days. In this session, the mice were placed in the pool facing the wall in one of the four quadrants. When the mouse located the platform, the time required to find the hidden escape platform (escape latency) was recorded and the mouse was allowed to stay on the platform for $15 \mathrm{~s}$. If the mouse did not locate the platform within $90 \mathrm{~s}$, it was placed on the platform for $15 \mathrm{~s}$ to familiarize it and the escape latency was considered to be $90 \mathrm{~s}$. On day 6, the probe trial was performed. In this session, the platform was removed. The time spent in the target quadrant and the number of times that the mouse crossed the removed platform was recorded and analyzed during $90 \mathrm{~s}$.

4.7. Tissue Processing. After the MWM test, the animals ( $n=6 /$ group) were decapitated and the brain was rapidly moved on ice. Thereafter, each mouse brain was weighed, homogenized in RIPA lysis buffer (with 1:100 PMSF, a protease inhibitor) to a concentration of $100 \mathrm{mg} / \mathrm{mL}$, and centrifuged at $10000 \mathrm{rpm}$ at $4{ }^{\circ} \mathrm{C}$ for $15 \mathrm{~min}$. The supernatants were collected and stored at $-80{ }^{\circ} \mathrm{C}$ for ELISA.

In addition, another cohort $(n=6 /$ group $)$ of mice was anesthetized with $5 \%$ chloral hydrate and transcardially perfused with a $0.9 \%$ saline solution, followed by $4 \%$ paraformaldehyde ( $\mathrm{pH}$ 7.4). The brains were removed from the skull and then fixed in the same $4 \%$ paraformaldehyde solution at $4{ }^{\circ} \mathrm{C}$.

4.8. Detection of the Levels of TNF- $\alpha$, IL-1 $\beta$, and IL- 6 by ELISA. The levels of TNF- $\alpha$, IL- $1 \beta$, and IL- 6 in the mice 
brains were assessed using ELISA kits (Boster, China) according to the manufacturer's instructions. Absorbance was read at $450 \mathrm{~nm}$ on a microplate spectrophotometer (BioTek). All the results are expressed as $\mathrm{pg} / \mathrm{mg}$.

4.9. Hippocampal Morphology Examination. To observe the histological changes in the hippocampus in the LPS-induced mice, the brain tissues were fixed in $4 \%$ paraformaldehyde solution at $4{ }^{\circ} \mathrm{C}$ for $24 \mathrm{~h}$. The brain specimens were paraffin-embedded and coronal sections were cut $(4 \mu \mathrm{m}$ thick) before mounting on silane-coated slides. The coronal sections were processed for HE staining according to a previously described method. ${ }^{32}$ The images were taken using an optical microscope (Olympus BX53F, Tokyo, Japan) at magnifications of $200 \times$ and $400 \times$, respectively.

4.10. Statistical Analysis. All the data are expressed as mean \pm standard deviation (SD). Statistical analysis was done by one-way analysis of variance followed by the StudentNewman-Keuls test using GraphPad Prism version 5.0 (GraphPad software, La Jolla, CA). $P$ values less than 0.05 were considered statistically significant.

\section{ASSOCIATED CONTENT}

\section{S Supporting Information}

The Supporting Information is available free of charge on the ACS Publications website at DOI: 10.1021/acsomega.8b02250.

HPLC chromatogram of standard compounds and their mixture; UV spectrum of each standard compound; the chemical structures of the identified compounds; the standard curves of NC and CA and the concentration and peak area of $\mathrm{NC}$ and $\mathrm{CA}$; the time line of drug treatment and experimental schedule (PDF)

\section{AUTHOR INFORMATION}

\section{Corresponding Author}

*E-mail: fanpeihong@sdu.edu.cn.

\section{ORCID $\odot$}

Hongxiang Lou: 0000-0003-3300-1811

Peihong Fan: 0000-0001-5529-8922

\section{Notes}

The authors declare no competing financial interest.

\section{ACKNOWLEDGMENTS}

This study was supported by the National Natural Science Foundation of China (Grant No. 81473323) and the Key R\&D Program in Shandong Province (No. 2015GSF119025).

\section{ABBREVIATIONS}

CA, cannabisin A; ELISA, enzyme-linked immunosorbent assays; HE, hematoxylin-eosin staining; HPLC, high-performance liquid chromatography; LPS, lipopolysaccharide; MWM, Morris water maze; NC, N-trans-caffeoyltyramine; TPA, total phenylpropanamides

\section{REFERENCES}

(1) Russo, E. B. History of cannabis and its preparations in saga, science, and sobriquet. Chem. Biodiversity 2007, 4, 1614-1648.

(2) Radwan, M. M.; ElSohly, M. A.; El-Alfy, A. T.; Ahmed, S. A.; Slade, D.; Husni, A. S.; et al. Isolation and pharmacological evaluation of minor cannabinoids from high-potency Cannabis sativa. J. Nat. Prod. 2015, 78, 1271-1276.
(3) Sarmento, L., Carus, M., Grotenhermen, F., Kruse, D.Scientifically sound guidelines for THC in food in Europe. http://eiha.org/media/2015/08/15-07-24-Report-Scientifically-SafeGuidelines-THC-Food-nova-EIHA.pdf, (accessed Nov 12, 2015).

(4) Montserrat-de la Paz, S.; Marín-Aguilar, F.; García-Giménez, M. D.; Fernández-Arche, M. A. Hemp (Cannabis sativa L.) seed oil: analytical and phytochemical characterization of the unsaponifiable fraction. J. Agric. Food Chem. 2014, 62, 1105-1110.

(5) House, J. D.; Neufeld, J.; Leson, G. Evaluating the quality of protein from hemp seed (Cannabis sativa L.) products through the use of the protein digestibility-corrected amino acid score method. J. Agric. Food Chem. 2010, 58, 11801-11807.

(6) Small, E.; Marcus, D. Hemp: a new crop with new uses for North America. In Trends in New Crops and New Uses; ASHS Press: Alexandria, 2002; pp 284-326.

(7) Mihoc, M.; Pop, G.; Alexa, E.; Radulov, I. Nutritive quality of romanian hemp varieties (Cannabis sativa $\mathrm{L}$.) with special focus on oil and metal contents of seeds. Chem. Cent. J. 2012, 6, 122-134.

(8) Cheng, C. W.; Bian, Z. X.; Zhu, L. X.; Wu, J. C. Y.; Sung, J. J. Y. Efficacy of a Chinese herbal proprietary medicine (hemp seed pill) for functional constipation. Am. J. Gastroenterol. 2011, 106, 120-129.

(9) Rodriguez-Leyva, D.; Pierce, G. N. The cardiac and haemostatic effects of dietary hempseed. Nutr. Metab. 2010, 7, 32-41.

(10) Frassinetti, S.; Moccia, E.; Caltavuturo, L.; Gabriele, M.; Longo, V.; Bellani, L.; et al. Nutraceutical potential of hemp ( Cannabis sativa L.) seeds and sprouts. Food Chem. 2018, 262, 56-66.

(11) Chen, T.; He, J. F.; Zhang, J. C.; Li, X. H.; Zhang, H.; Hao, J. $\mathrm{X}$.; Li, L. The isolation and identification of two compounds with predominant radical scavenging activity in hempseed (seed of Cannabis sativa L.). Food Chem. 2012, 134, 1030-1037.

(12) Pojić, M.; Mišan, A.; Sakač, M.; Dapčević Hadnađev, T.; Šarić, B.; Milovanović, I.; Hadnađev, M. Characterization of byproducts originating from hemp oil processing. J. Agric. Food Chem. 2014, 62, 12436-12442.

(13) Lin, Z. M.; Chen, L. M.; Liang, Z. M.; Xia, X. The effect of different extractants from hemp seed in mice with <tepcommon:author-query $>\mathrm{AQ} 2$ : Please provide a DOI number for ref 13 or indicate if one doesn\&amp;\#x2019;t exist.</tep-common:author-query>experimental Alzheimer's disease. Pharmacol. Clin. Chin. Materia Medica 2016, 32, 130-134.

(14) Luo, J.; Yin, J. H.; Wu, H. Z.; Wei, Q. Extract from Fructus cannabis activating calcineurin improved learning and memory in $<$ tep-common:author-query $>\mathrm{AQ} 3$ : Please provide a $\mathrm{DOI}$ number for ref 14 or indicate if one doesn\&amp; \#x2019;t exist.</tepcommon:author-query $>$ mice with chemical drug-induced dysmnesia. Acta Pharmacol. Sin. 2003, 24, 1137-1142.

(15) Luo, Q.; Yan, X. L.; Bobrovskaya, L.; Ji, M.; Yuan, H. Q.; Lou, H. X.; Fan, P. H. Anti-neuroinflammatory effects of grossamide from hemp seed via suppression of TLR-4-mediated NF- $\kappa$ B signaling pathways in lipopolysaccharide-stimulated BV2 microglia cells. Mol. Cell. Biochem. 2017, 428, 129-137.

(16) Yan, X.; Tang, J. J.; Passos, C. D.; Nurisso, A.; Simoes-Pires, C. A.; Ji, M.; Lou, H. X.; Fan, P. H. Characterization of lignanamides from hemp (Cannabis sativa L.) seed and their antioxidant and acetylcholinesterase inhibitory activities. J. Agric. Food Chem. 2015, 63, 10611-10619.

(17) Zhou, Y. F.; Wang, S. S.; Lou, H. X.; Fan, P. H. Chemical constituents of hemp (Cannabis sativa L.) seed with potential antineuroinflammatory activity. Phytochem. Lett. 2018, 23, 57-61.

(18) Lemoine, L.; Saint-Aubert, L.; Nennesmo, I.; Gillberg, P. G.; Nordberg, A. Cortical laminar tau deposits and activated astrocytes in Alzheimer's disease visualised by 3H-THK5117 and 3H-deprenyl autoradiography. Sci. Rep. 2017, 7, No. 45496.

(19) Cunningham, C. Microglia and neurodegeneration: the role of systemic inflammation. Glia 2013, 61, 71-90.

(20) Frühauf, P. K.; Ineu, R. P.; Tomazi, L.; Duarte, T.; Mello, C. F.; Rubin, M. A. Spermine reverses lipopolysaccharide-induced memory deficit in mice. J. Neuroinflammation 2015, 12, 3. 
(21) Solomon, U.; Taghogho, E. A. Methyl jasmonate attenuates memory dysfunction and decreases brain levels of biomarkers of neuroinflammation induced by lipopolysaccharide in mice. Brain Res. Bull. 2017, 131, 133-141.

(22) Elsohly, M. A., Radwan, M. M., Gul, W., Chandra, S., Galal, A. Phytochemistry of Cannabis sativa L. https://link.springer.com/ content/pdf/10.1007\%2F978-3-319-45541-9.pdf, 2017, 103, 1-36.

(23) Vanhoenacker, G.; Van Rompaey, P.; De Keukeleire, D.; Sandra, P. Chemotaxonomic features associated with flavonoids of cannabinoid-free Cannabis (Cannabis sativa subsp. sativa L.) in relation to hops (Humulus lupulus L.). Nat. Prod. Lett. 2002, 16, 5763.

(24) Yan, X. L.; Zhou, Y. F.; Tang, J. J.; Ji, M.; Lou, H. X.; Fan, P. H. Diketopiperazine indole alkaloids from hemp seed. Phytochem. Lett. 2016, 18, 77-82.

(25) Sakakibara, I.; Ikeya, Y.; Hayashi, K.; Mitsuhashi, H. Three phenyldihydronaphthalene lignanamides from fruits of Cannabis sativa. Phytochemistry 1992, 31, 3219-3223.

(26) Sakakibara, I.; Ikeya, Y.; Hayashi, K.; Okada, M.; Maruno, M. Three acyclic bis-phenylpropane lignanamides from fruits of Cannabis sativa. Phytochemistry 1995, 38, 1003-1007.

(27) Sakakibara, I.; Katsuhara, T.; Ikeya, Y.; Hayashi, K.; Mitsuhashi, H. Cannabisin A, an arylnaphthalene lignanamide from fruits of Cannabis sativa. Phytochemistry 1991, 30, 3013-3016.

(28) Cai, P.; Fu, X.; Deng, A. G.; Zhan, X. J.; Cai, G. M.; Li, S. X. Anti-aging effect of hemp seed oil, protein and <tep-common:authorquery $>A Q 4$ : Please provide a DOI number for ref 28 or indicate if one doesn\&amp;\#x2019;t exist.</tep-common:author-query>lignanamide on old mice. Cent. South Pharm. 2010, 8, 165-170.

(29) Kurz, C.; Ungerer, I.; Lipka, U.; Kirr, S.; Schütt, T.; Eckert, A.; et al. The metabolic enhancer piracetam ameliorates theimpairment of mitochondrial function and neuriteoutgrowth induced by $\beta$-amyloid peptide. Br. J. Pharmacol. 2010, 160, 246-257.

(30) Tripathi, A.; Paliwal, P.; Krishnamurthy, S. Piracetam attenuates LPS-induced neuroinflammation and cognitive impairment in rats. Cell. Mol. Neurobiol. 2017, 37, 1373-1386.

(31) Vorhees, C. V.; Williams, M. T. Morris water maze: procedures for assessing spatial and related forms of learning and memory. Nat. Protoc. 2006, 1, 848-858.

(32) Wang, P. P.; Sun, H. X.; Liu, C. J.; Hu, M. H.; He, X. Q.; Yue, S.; Jiao, Z. Z.; Xiang, L. Racemic oleracein $\mathrm{E}$ increases the survival rate and attenuates memory impairment in $\mathrm{D}$-galactose/NaNO2-induced senescent mice. Phytomedicine 2016, 23, 460-467. 